\title{
Analysis of the Intelligent Form of Traditional Art Exhibitions from the Perspective of Digital Media
}

\author{
Xiangying Chen \\ School of Communication \\ Zaozhuang University \\ Zaozhuang, China 277100
}

\begin{abstract}
In the context of the integration of intelligent technologies and digital media, in this paper, the author has studied the intelligent form of traditional art exhibitions, taken the classic cases and development status of intelligent exhibitions at home and abroad into account, and sorted out the technical means, implementation methods, social influences and prospects of digital media in the spread and display of traditional arts, to provide ideas and suggestions for the better spread, inheritance and transformation of traditional arts.
\end{abstract}

\section{Keywords — digital media; traditional arts; exhibitions}

\section{INTRODUCTION}

Due to the original intention of protecting rare cultural relics and the high costs of transportation, borrowing, insurance, etc., the time and place of exhibitions of traditional art works at home and abroad are greatly limited. In recent years, with the constant development of digital media, more media tools have been used in the exhibitions of classical arts, such as the dynamic images, interactive devices, and immersive art and so on. Through the exhibitions based on digital media which are replacing exhibitions displaying material objects, people can know more about the real looks of the artworks in the round by acquiring multidimensional perceptions of the artworks. That is "the content is the medium". This form of exhibition has closed the distance between ordinary persons and the classics and become an intelligent spread form of arts. The immersive digital sound and picture display project of the Palace Museum named "Going into the picture of riverside scene at Qingming Festival" breaks the traditional way to hang the picture for display, and the digital touch screen is used to present videos, providing visitors with excellent audio-visual feelings. The audience only needs to touch certain parts on the screen to get corresponding details of the scroll that they are interested in, obtaining the diversified experience of seeing, hearing and touching. The "Qianlong upsurge" exhibition of the Palace Museum in Taipei enables the audience to experience the Qing culture and appreciate the royal artifacts through various entertaining interactive devices; The art exhibition named "Immortal Van Gogh" used multimedia animation technology to make the vivid and intense colors in Van Gogh's works flow and rotate on the screen, attracting visitors to stop in front of the screens and interact with them; Jean Renoir global tour has adopted the current popular VR virtual reality interactive technology to make the audience feel more immersive and get the mysterious charms of the works.

Through the intelligent media forms and all-around perceptions, people can have a direct understanding of the real looks of the artworks. Even if they have not seen the original works, they can still have a comprehensive understanding of them and feel their charms.

\section{The ApplicAtion of Digital Media TeChNOLOGY IN TRADITIONAL ART EXHIBITIONS}

Regardless of whether it is accepted, the impact of new technologies and ideas such as cloud computing, mobile internet, Internet of Things, and big data intelligent constructions on traditional cultures has appeared. Museums of material objects and digital museums are changing and transforming to smart museums and pan-museums.

In the exhibitions of traditional art works, the intelligent displays are not limited to the traditional methods such as "hanging artworks on the wall". The intelligent exhibitions are no longer simple displays for the artwork information, but are turning to the reveal and service of the cultural information with art as the core.

\section{A. Introduction to the Multimedia Information}

Take the touch screen as the basic carrier, and achieve stereoscopic display and scene restoration through processes of digital acquisition, digital modeling, 3D animation, etc., making the exhibits more attractive and appealing; [1] use the multimedia means such as electronic pictures and words, animated videos, immersive theaters, etc. to integrate the words, images of works, sound, even scent and touch, and to deeply interpret the stories behind the works, giving the audience a controllable all-dimensional stereoscopic cognition.

For example, the British Museum has scanned the eight mummies in the collection with the most advanced medical scanning equipment, and then displayed the CT images from the scanning in the exhibition. Visitors can see the mummy scans "layer by layer" on the screen by rotating the knob on the interactive device, thus getting the feeling of uncovering the mummy wraps with their own hands. 


\section{B. Natural User Interface}

The so-called natural user interface, is that the computer gets the information of human gestures, body movements, expressions, voices, sounds, and other environmental factors, and replaces the keyboard and mouse to achieve humancomputer interaction. For example, the "Qianlong upsurge" device exhibition curated by the Palace Museum in Taipei has used this technology: the image of Emperor Qianlong in the mirror will make corresponding reactions according to people's standing positions, movements and expressions, which can instantly stimulate people's interest in the exhibition. According to the color specimens collected by the cameras from the visitors, different paintings of porcelain artifacts can be drawn, which is very impressive. In the 2016 art exhibition named "The Drifting Civilization" held in the China Millennium Monument, this technique was used to show the "Venus with No Arms" (also known as Venus of Milo). If the visitors stand at the designated position and make different actions with their arms, the Venus statue of 1:1 scale on the screen will grow arms in different shapes. Using the technologies of natural user interface makes the interaction between people and computer more positive and deep. The human-object-digital display model is further developed and transforming into the intelligent display mode, and the content of exhibitions is effectively expanded and becoming more immersive.

\section{Games}

The games based on interactive devices in classical art exhibitions can be implemented in various forms. Visitors can operate the devices and play games in accordance with certain rules, and they will get information about the exhibits and interesting sensory experiences in entertainment. For example, in the "Qianlong upsurge" exhibition, people can step on the projection on the ground to interact with the projector equipment so as to get the projected patterns changed and obtain relevant information about the exhibits. For the video installation that introduces the court life, the visitors can take head shots of themselves through the camera, and then the system captures and uploads the shots to make them appear in the cartoon film on the screen as the characters of the film, which is very impressive.

\section{Holographic Images and Virtual Reality}

Holographic technology is a technique that can record and reproduce the real three-dimensional image of an object basing on the principles of interference and diffraction. [2] The holographic image device has been widely used in the exhibitions of cultural relics, and it can fully and vividly reproduce the information about the cultural relics from all 360 degrees, and the color and texture presented is almost the same as that of the original artworks. In addition, the virtual reality technology can also be used to reproduce the reality. Visitors can use sensor tracking devices such as helmet displays, data gloves, and somatosensory vests to interact with the visual world produced by the equipment with their vision, auditory sense and other sensations, feeling much dedicated and immersed in the virtual space. [3] For example, in the "University Public Welfare Tour of Fine Artworks of
Dunhuang frescoes" launched nationwide by the Dunhuang Academy and China Dunhuang Grottoes Conservation Research Foundation, visitors can virtually roam more than 30 rare and precious caves through the VR technology. With VR glasses, visitors can freely turn their bodies to view various parts of the caves, and can enlarge the part of murals in their sight through controlling their point of sight to achieve close observation.

\section{E. AR: Augmented Reality Technology}

Augmented Reality (AR) is a technology that calculates the position and angle of camera images in real time and adds corresponding images. The goal of this technology is to set the virtual world in the real world and make them interact with each other on the screen. [4] Augmented reality further increases the derivative value of exhibits and expands the range of sensations of visitors.

\section{The SignificAnce of Digital Media TeChNOLOGY TO TRADITIONAL ART EXHIBITIONS}

The forms of traditional art are diverse and the content of it is magnificent. The inheritance, transformation and development of traditional art are of vital importance to the construction of modern socialist spiritual civilization and the establishment of harmonious values. The intelligent spread mode under the context of "smart +" combines the latest intelligent technology with precise information interaction design, bringing people extrasensory special experiences. The sense of immersion, fun, degree of recognition, memory intensity and artistic conversion probability of the intelligent spread mode are all higher than those of the traditional way of information dissemination used in the past. It is meaningful to apply the new mode to relevant categories of traditional art on the basis of the researches and summaries about it.

\section{A. The Era of All-media Hybrid Stereo Interpretation Comes}

Due to the convenience, reproducibility and interactivity of digital media technology, the cost of traditional art exhibitions is greatly reduced and the digital media has a huge propagation effect. Audiences of all ages understand and experience the traditional art works in this medium form that is different from the form of the past, and participate in the new mode with enthusiasm, which brings more extensive derivative meanings to the classical arts.

Through the new forms of perception created by the cutting-edge digital media technologies, the audience itself has been made part of the contemporary traditional art exhibitions. The effects of the exhibition presented will be different according to the different reactions and operations of visitors during the visiting process. These extrasensory special experiences are the true results of the deep interpretation of various technologies such as multimedia technology, natural interface interaction and virtual reality.

\section{B. Easing the Interpretation, Fragmenting the Content and Replacing Sublimity with Novelty}

In the context of intelligent technologies, various media in forms of texts, pictures, images, sounds, smells, temperature, 
touch, etc. is integrated and applied to the exhibitions of traditional arts. People can know about the real looks of artworks through various media of different forms. "Content is the medium", based on which more interactive devices are applied to the spread and display of traditional arts. For example, a set of virtual reality equipment can make the audience feel the charm of Dunhuang murals in an immersive way, the effect of which is far more excellent than only watching a movie or several pictures on the Internet. The extensiveness, precision, fun and profoundness of this mode of communication will greatly promote the inheritance and development of traditional arts.

In addition, as a gimmick to promote marketing, new media technology can easily become just a means of the business to make profits. In some traditional art exhibitions, the holders only pursue the novel and entertaining effect to attract the audience. On the surface, there seems to be much interactive experience feedback, but the form and content of the exhibition cannot coordinate the sublimity and fun of traditional arts. It is contrary to the original intention and cannot present the real charm and value of artworks, becoming just a vulgar means to make profits. The use of intelligent and digital technology should be rationalized, and the intelligent dissemination in form and content of traditional art should be adhered to, and the breadth and depth of dissemination of traditional art in the audience should be improved.

\section{Big Data Processing and Dynamic Visualized \\ Presentation Are Realized and the Dissemination Mode Is Transforming to the Intelligent Type}

In addition to having inherited the essential characteristic of "communication", the intelligent dissemination method for art also applies cutting-edge technologies, shows good conceptions and interacts well with visitors, which makes it richer in content and more diversified in forms than the traditional dissemination means of art. [5]The cutting-edge sciences and technologies and the attractive mode of exhibition make art exhibitions in intelligent modes stand out in today's urban culture. Through the most advanced technologies of digital media, the new mode has broken through the materiality and unitary of the previous exhibition mode, which brings new meanings to the exhibition projects.

\section{Transformation to Popular Trends Under the Intelligent Dissemination Mode}

The dissemination methods of traditional art supported by intelligent technologies are usually combined with various audiovisual art forms such as images, music and animation to interact better with visitors. Compared with other modes of dissemination, it is more immersive and interesting, which is conducive to the popularity transformation of traditional art and the formation of inheritance and innovation ways combining with the popular cultural economy.

The "smart +" dissemination model combines multiple advanced technologies and in-depth interpretation forms, and comprehensively applies technical means such as the multimedia technology, natural interface interaction, and virtual reality to display the artworks from multiple angles.
The new perceptions and feelings created by the cutting-edge technologies are combined with the popular elements, and audiovisual elements such as music, images and animation are integrated to give visitors an unprecedented extrasensory experience. This provides a new form of perception for the audience, and the efficiency of communication and interaction has also been greatly improved, and even the audience has become part of the exhibition. Many of the exhibition projects have presented different interaction effects according to the different reactions and operations of the participants, which has created and interpreted new meanings for arts. Based on this, the "smart + " dissemination model can greatly promote the transformation to popular trends of traditional art and further construct the integration of traditional art and economy.

\section{CONCLUSION}

Classical art masters communicate with people through their works across the long time river between them. With their unparalleled charm and wisdom, classical artworks lead the moderns living in reinforced concrete buildings and information explosion to introspect themselves, observe the truth and kindness and look directly at their souls.

With the rapid development of technologies, the classical art exhibition displayed with new media is becoming a popular trend, which is unstoppable. But are the virtual illusions the final destination and ultimate expression of classical art? Before the day when the virtual illusions and reality are integrated, how should we face the innovations and impacts of new media on the dissemination of classical art?

The focus of the exhibitions in intelligent forms should still be on the exhibits themselves, not on the technical means. The understanding of the content of the exhibition, as well as the master and control of the audience's psychology and the environment of the exhibition hall, is still the source of design concepts for the exhibition, and is still the key to success of the exhibition.

How to make the collections and research results on the "top shelf" more approachable for the public, further reveal the connotation of cultural information, and realize the value of traditional arts to service society, thus further reflecting the value of museums as the social service organizations is a question that should be thought about in quite a few current traditional art exhibitions. The practical issues that the digital media intelligent means is facing are still the problems of how to "hold the exhibition from the practical point of view of the exhibits, take serving the audience as the core, and continuously apply advanced technologies". Currently, further deepening the contents of exhibitions and improving the performance is the only way for museums to realize their own value.

\section{REFERENCES}

[1] Zheng Weili. The changes of role andfunctions of museums in the era of new media[J], Film Museum • Impact (2017, Issue 02, Total Issue 110), 2017-04-01. (in Chinese)

[2] Sun Xiaohong. 3D display is coming to "Carnival" [J], China Internet Week, 2011-02-20. (in Chinese) 
[3] Wang Yuan. Thoughts on the Application of China Comic and Animation Museum Based on VR Technology [J], Popular Literature, 2017-04-30. (in Chinese)

[4] $\mathrm{Su}$ Yushan. Innovations in Interactive Advertising Design in Digital Media [D], Beijing, North China University of Technology, 2018-04-05 (in Chinese)

[5] Ma Xiaoxiang. Domestic New Media Art Exhibitions and the Development of New Media Art Disciplines in Colleges and Universities[J], Journal of Nanjing University of the Arts (Art and Design), 2010-12-15. (in Chinese) 